\title{
Baculovirus as versatile vectors for protein expression in insect and mammalian cells
}

\author{
Thomas A Kost ${ }^{1}, \mathrm{~J}_{\text {Patrick Condreay }}{ }^{1} \&$ Donald L Jarvis ${ }^{2}$
}

\begin{abstract}
Today, many thousands of recombinant proteins, ranging from cytosolic enzymes to membrane-bound proteins, have been successfully produced in baculovirus-infected insect cells. Yet, in addition to its value in producing recombinant proteins in insect cells and larvae, this viral vector system continues to evolve in new and unexpected ways. This is exemplified by the development of engineered insect cell lines to mimic mammalian cell glycosylation of expressed proteins, baculovirus display strategies and the application of the virus as a mammalian-cell gene delivery vector. Novel vector design and cell engineering approaches will serve to further enhance the value of baculovirus technology.
\end{abstract}

Over the past 20 years the baculovirus-insect cell expression system has become one of the most widely used systems for routine production of recombinant proteins ${ }^{1-6}$. A number of technological improvements have eliminated the original tedious procedures required to identify and isolate recombinant viruses, increasing the popularity of the system. These include development of a wide variety of transfer vectors, simplified recombinant virus isolation and quantification methods, advances in cell culture technology and the commercial availability of reagents. These enhancements have resulted in a virus-based expression system that is safe, easy to use and readily amenable to scale-up.

In addition, biotechnology now uses baculoviruses in applications beyond the production of proteins in insect cells and larvae. These include the development of strategies for displaying foreign peptides and proteins on virus particles and the insertion of mammalian cellactive expression cassettes in baculoviruses to express genes efficiently into many different mammalian cell types. Baculoviruses engineered to display foreign peptides and proteins on the viral surface have proven particularly useful as immunogens and both surface display and capsid fusions may provide further opportunities for enhancing and targeting baculovirus-mediated transduction of mammalian cells.

Here, we review recent advances in baculovirus-insect cell protein production, baculovirus display and the development and application of baculoviruses as mammalian-cell gene-delivery vectors (Fig. 1).

\section{Isolation and quantification of recombinant baculoviruses}

Recombinant baculovirus expression vectors were initially isolated using a highly inefficient homologous recombination process. Insect cells cotransfected with baculovirus and transfer plasmid DNA produced a mixture of parental and recombinant viruses, with a recombination frequency of only about $0.1 \%$. Progeny were usually resolved by

${ }^{1}$ Gene Expression Protein Biochemistry, GlaxoSmithKline R\&D, 5 Moore Drive, Research Triangle Park, North Carolina 27709, USA. 2Department of Molecular Biology, University of Wyoming, Laramie, Wyoming 82071, USA. Correspondence should be addressed to T.A.K. (tom.a.kost@gsk.com).

Published online 5 May 2005; doi:10.1038/nbt1095 plaque assay and recombinant clones identified microscopically by their distinctive occlusion-negative plaque phenotypes. This was tedious as recombinant plaques, surrounded by a sea of occlusion-positive parental virus plaques, were difficult to identify. A huge improvement came with the development of baculovirus DNA that could be linearized at a unique Bsu36I site in the polyhedrin locus ${ }^{7}$. When used together with a transfer plasmid to cotransfect insect cells, the linearized viral DNA gave rise to recombinants at a higher frequency, typically around 25\%. Later, baculovirus DNAs were engineered to have multiple Bsu36I sites, one within an essential viral gene ${ }^{8}$. Bsu36I digestion created a large deletion that functionally inactivated the essential gene, thus precluding replication of parental virus and increasing the frequency of recombinant virus production to over $90 \%$. This approach was commercialized and the use of predigested viral DNAs became status quo for recombinant baculovirus production. Still, baculovirus plaque assays remained an essential part of the technology, as recombinant baculoviruses were most frequently cloned using this approach.

Efforts to eliminate the requirement for a plaque assay in virus isolation led to development of an in vivo bacterial transposition method, first described in 1993 and later commercialized as the Bac-to-Bac system $^{9}$. This method involves site-specific transposition of a foreign gene from a donor plasmid to a cloned baculoviral DNA, or 'bacmid' such that the foreign gene is controlled by the polyhedrin promoter. Since Escherichia coli clones containing recombinant bacmid DNA acquire an antibiotic resistance marker and lose a lac $Z$ marker, they can be easily selected and identified. One simply isolates viral DNA from positive bacterial clones and uses this bacmid DNA to transfect insect cells and produce recombinant virus. Theoretically this method does not require a plaque assay to resolve parental from recombinant virus progeny; however, the virus stock may be, nevertheless, polyclonal. A recent improvement in the frequency of recombinant viruses produced using Tn7-mediated transposition has been described, which may allow for the efficient generation of baculoviral libraries ${ }^{10,11}$. It is important to note that recent reports have shown that BAC vector sequences can be spontaneously excised from bacmid-derived vectors upon passage in insect cells ${ }^{12,13}$. In our experience, this has not posed a major problem 


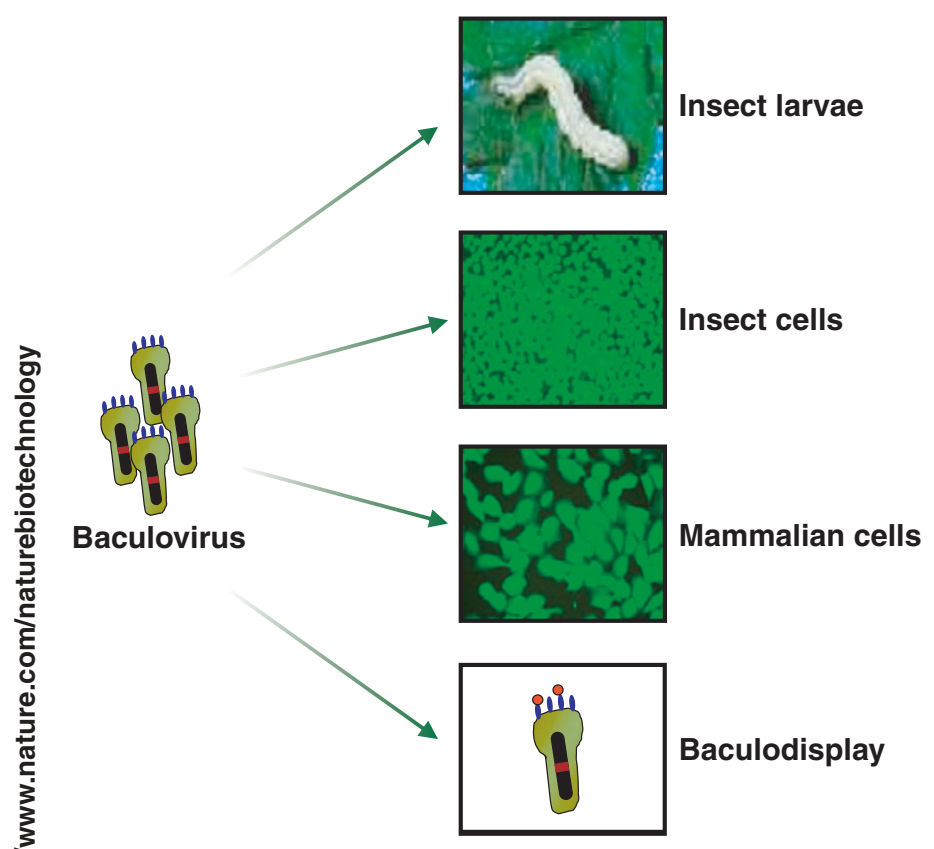

Figre 1 Versatility of baculovirus expression vectors. Baculovirus vectors can be used for a variety of applications. These include producing proteins in insect larvae, insect cells and mammalian cells. The insect and mammalian cells in the photomigrographs were treated with baculoviruses expressing GFP. Viruses can also be produced that display peptides or proteins on the surface of viral particles. The red circles on the schematic virus particle represent displayed gp64 fusion proteins.

for routine protein production using bacmid-derived viruses, but could hinder large-scale production systems.

Recently two additional approaches have been described for generating recombinant baculoviruses. An in vitro transposition system, adapted from Bac-to-Bac, commercially known as BaculoDirect, uses a purified transposase to move a foreign gene from a donor plasmid to a viral DNA acceptor, placing the foreign gene under the control of the polyhedrin promoter. The parental viral DNA has a herpes virus thymidine kinase gene, which serves as a negative selection marker that is eliminated upon transposition. Thus, insect cells are transfected with the mixture of parental and recombinant viral DNA created in the test tube and parental viruses are eliminated by gancyclovir treatment. As with Bac-to-Bac, plaque isolation is not required, but the virus stock may be polyclonal and an experienced virologist would probably undertake plaque purification. An alternative method uses a baculovirus with a lethal mutation in orf1629, which encodes an essential gene product required for virus replication. Viral DNA is maintained as a bacmid in E. coli. In this system cotransfection of insect cells with a transfer vector containing the gene of interest and the engineered viral DNA yields $100 \%$ recombinant viruses ${ }^{14}$.

Studies have also been undertaken to develop rapid and facile methods for virus quantification. These efforts have been driven by the use of automated platforms for baculovirus production and expression ${ }^{15-17}$ and the need to rapidly titrate baculovirus stocks destined for the production of multi-subunit protein complexes and mammalian cell transduction studies. A commercially available immunocytochemical assay using a monoclonal antibody against the baculovirus gp64 protein provides virus titers within $48 \mathrm{~h}^{18}$. Two additional antibody-based assays have been developed that provide rapid virus titrations ${ }^{19,20}$. Quantification of baculovirus particles by flow cytometry ${ }^{21}$ and real-time PCR $^{22}$ has been described. Several assays that use reporter proteins such as green fluorescent protein (GFP) or $\beta$-galactosidase have also been described; however, an inherent drawback of these methods is the need for expression of an additional protein.

\section{Protein glycosylation in the baculovirus- insect cell system}

It is often stated that the baculovirus-insect cell system has eukaryotic protein processing capabilities. It is generally true that insect cells can fold, modify, traffic and assemble newly synthesized polypeptides to produce highly authentic, soluble end products ${ }^{1,2,23}$. However, it is equally true that insect protein processing pathways are not necessarily equivalent to those of higher eukaryotes. One of the best examples of a similar, but distinct processing pathway is the protein $N$-glycosylation pathway.

Early research on dipteran insect cells, which has been reviewed in detail elsewhere ${ }^{24,25}$, established a model of the insect protein $N$-glycosylation pathway which, with a few caveats, is still valid today. These studies indicated that insect cells could assemble $N$-glycans, transfer them to nascent polypeptides and trim the $N$-glycan precursors to produce high mannose or paucimannose end products (Fig. 2). However, the cells failed to elongate the trimmed $\mathrm{N}$-glycans to produce complex products containing terminal galactose and/or sialic acid residues. As recombinant glycoproteins began to be produced, it was recognized that the lepidopteran insect cell lines used as hosts for baculovirus expression vectors followed this general paradigm. In addition, enzyme assays showed that these cell lines had little or none of the galactosyltransferase and sialyltransferase activities involved in $\mathrm{N}$-glycan elongation (Fig. 2). Moreover, it was found that many of these cell lines have an unusual processing activity that converts an intermediate common to both the insect and mammalian pathways to the insect-specific paucimannose end product ${ }^{26}$ (Fig. 2).

Today, it is generally recognized that most baculovirus-expressed recombinant glycoproteins will acquire authentic $N$-glycans only at sites occupied by high mannose structures on the native mammalian products. In contrast, they are most likely to acquire paucimannose $N$ glycans at sites occupied by complex, terminally galactosylated and/or sialylated $\mathrm{N}$-glycans on the native product. This latter fact is a clear limitation of the baculovirus-insect cell expression system because $N$-glycans, and particularly terminal sialic acids, contribute to glycoprotein functions in many different ways. For some clinical applications, such as in vivo administration of a therapeutic recombinant glycoprotein, the absence of terminal sialic acids would be unacceptable.

Recent trends in the development of the baculovirus-insect cell system include extensive efforts to address this problem, the details of which have been reviewed elsewhere ${ }^{27-31}$. However, an overview of selected developments will be of interest to investigators using the baculovirus-insect cell system for recombinant glycoprotein production. An early step was the development of expression plasmids and methods for transforming lepidopteran insect cell lines containing stably integrated, constitutively expressed foreign genes ${ }^{32,33}$. These studies set the stage for the creation of transgenic lepidopteran insect cell lines containing mammalian genes encoding $\mathrm{N}$-glycan processing activities that were absent in the parental cell lines. The first transgenic insect cell line of this type was produced by transformation with a bovine $\beta 1,4$-galactosyltransferase gene. Baculovirus infection of this cell line, but not the parental $S f 9$ cell line, led to the production of a foreign protein with terminally $\beta$-galactosylated $N$-glycans ${ }^{34}$. Subsequently, a transgenic $S f 9$ line encoding both bovine $\beta 1,4$-galactosyltransferase and rat $\alpha 2,6$-sialyltransferase was isolated that supported the production of terminally $\alpha 2,6$-sialylated $N$-glycans ${ }^{35}$. This was a surprising result because the donor substrate required by the rat sialyltransferase, CMP-sialic acid, is not found at detectable levels in $S f 9$ 
cells $^{36,37}$. A possible explanation was obtained with the finding that $S f 9$ cells have a sialic acid scavenging pathway that can support de novo glycoprotein sialylation in transgenic cells expressing the sialyltransferase $\mathrm{e}^{38}$. However, detailed structural analyses revealed that only the lower $(\alpha 1,3)$ branch of the $N$-glycans produced by these transgenic insect cells had been elongated. Because monoantennary $N$-glycans are rarely found on mammalian glycoproteins, another transgenic insect cell line was designed to express five mammalian glycosyltransferases, including one that initiates elongation of the upper $(\alpha 1,6) N$-glycan branch ${ }^{39}$. These cells, designated SfSWT-1 cells produced biantennary, terminally monosialylated $N$-glycans. Finally, because a sialic acid scavenging pathway might be an inefficient way to produce CMP-sialic acid for de novo glycoprotein sialylation, a transgenic line designated SfSWT-3 was produced. These cells encode the same five glycosyltransferases of SfSWT-1, plus two murine enzymes that convert the sialic acid precursor, $N$-acetylmannosamine, to CMP-sialic acid ${ }^{40}$. When cultured in the presence of $\mathrm{N}$-acetylmannosamine, this new cell line produced high levels of intracellular CMP-sialic acid and a recombinant

$N$-glycoprotein with a highly homogeneous, biantennary, monosialylated side chain.

The transgenic insect cell line approach has begun to address the inability of the baculovirus-insect cell system to produce authentic recombinant $\mathrm{N}$-glycoproteins; however, the humanization of protein processing pathways in insect cells is a work in progress, with many additional developments needed and yet to come.

\section{Enhancing protein expression in insect cells}

In many instances sufficient quantities of functional protein for experimental needs can be readily obtained from baculovirus-infected insect cells. However, this is not always true and for numerous reasons increased yields of functional protein are often desirable. Various approaches to increasing production of properly processed proteins were covered in an earlier review on this topic ${ }^{41}$. A number of studies have documented enhanced protein production following cotransfection with baculoviruses expressing chaperone proteins, which are known to aid in the folding and modification of newly synthesized proteins. The expression of correctly assembled Shaker potassium channels in $S f 9$ cells was enhanced by coexpression of the calcium-binding, lectin-chaperone calnexin together with substitution of the polyhedrin promoter with the weaker basic protein promoter to drive expression of the ion channel ${ }^{42}$. Coexpression of calreticulin promoted the production of properly folded human lipoprotein lipase ${ }^{43}$ and HLA-DR4 tetramers ${ }^{44}$. Another approach has been to coexpress the chaperone Hsp70 and its cofactors Hsdj and Hsp40. Such coinfections have resulted in increased yields of soluble Epstein-Barr virus replication protein, BZLF $1^{45}$ and functionally active tumor suppression protein $\mathrm{LKB} 1^{46}$. These studies demonstrate the potential value of coexpressing chaperones to enhance functional protein production.

Significant increases in expression levels have also been reported by the addition of various DNA elements to the virus. The addition of baculovirus homology region $1(h r 1)^{47}$ and $h r 3$ (ref. 48 ) sequence regions to the virus genome resulted in increased luciferase production. Incorporation of a 21-base-pair (bp) element derived from a $5^{\prime}$ untranslated leader sequence of a lobster tropomyosin cDNA that contains the Kozak sequence and A-rich sequence found in the polyhedrin leader sequence into a recombinant virus enhanced the expression of tropomyosin and luciferase 20- and sevenfold, respectively ${ }^{49}$. As with the addition of hr elements, the effect of this 21-bp element will require further evaluation with additional proteins to determine the general applicability of this approach for enhancing protein yield.

Baculovirus infection of insect cells results in microscopically observable cell lysis within 3-5 d after infection. Cell disruption may lead to increased proteolytic activity and other environmental factors that can result in degradation of recombinant protein. In an attempt to overcome this difficulty, a baculovirus with reduced capability for initiating cell lysis was isolated by random mutagenesis and the application of a novel fluorescence resonance energy transfer (FRET)-based assay for selecting the desired mutant ${ }^{50}$. At $5 \mathrm{~d}$ after infection the mutant virus showed only $7 \%$ lysis of infected $S f 21$ cells, whereas the parent virus showed $60 \%$ lysis. Using this virus the authors demonstrated that a higher level of compactly folded, engineered luciferase protein could be produced with less degradation as compared to the parental virus. Another approach to reducing protein degradation is the development of a chitinase and v-cathepsin negative bacmid. Generation of a recombinant virus designed to express the cattle parasite Theileria parva sporozoite surface protein p67 with this bacmid protected the secreted recombinant protein from degradation ${ }^{51}$.

The baculovirus-insect cell system has been used successfully for the expression of thousands of diverse types of proteins. It has proven particularly valuable for the expression of $G$ protein-coupled receptors (GPCRs) ${ }^{52,53}$ and coexpression with $G$ proteins has proved valuable for studying receptor-G protein interactions ${ }^{54}$. The system has also proven very useful for expression of cytochrome p450 enzymes ${ }^{55-57}$. Irrespective of the protein being produced, a major advantage of the baculovirus-insect cell expression system is the ease of scale-up from the laboratory to a large-scale production system ${ }^{58}$. 


\section{Production of virus-like particles (VLPs) and protein complexes}

The baculovirus-insect cell system has been used extensively for the production of VLPs to study viral assembly processes in the absence of infectious virus, produce antigens for immunization and proteins for diagnostic assays and for gene transfer ${ }^{59-62}$. This approach is particularly valuable in those cases where cell culture-based viral replication systems are not available, such as human papilloma virus (HPV) and hepatitis $\mathrm{C}$ virus (HCV). The baculovirus-insect cell system allows one to deliver individual viral structural proteins via coinfection with multiple baculoviruses, each expressing a single protein, or via a single virus designed to express multiple proteins ${ }^{63}$. By varying the multiplicity of infection and by using various promoters, one can attempt to control the amount of expressed proteins to optimize VLP production.

A striking example of the application of this technology is the development of HPV VLP-based vaccines ${ }^{64,65}$. VLPs composed of the HPV types $16 / 18 \mathrm{~L} 1$ structural proteins produced in baculovirus-infected insect cells have been shown in clinical trials to be efficacious in preventing cervical infections with HPV-16 and HPV-18, together with the associated cytological abnormalities and lesions ${ }^{66}$. HCV VLPs have also been successfully produced using the baculovirus-insect cell system ${ }^{67-71}$. VLPs containing the core, $\mathrm{E} 1$ and $\mathrm{E} 2$ proteins of $\mathrm{HCV}$ resemble putative $\mathrm{HCV}$ virions and have been shown to effectively induce HCV-specific humoral and cellular immune responses in baboons ${ }^{71}$. Recently the assembly of human severe acute respiratory syndrome (SARS) coronavirus-like particles in baculovirus-infected insect cells expressing the S, E and M structural proteins has been described ${ }^{72,73}$. Budded VLPs could only be detected in the culture medium when the genes encoding the three proteins were carried by a single recombinant baculovirus ${ }^{73}$. These results provide impetus for further studies into the assembly and development of candidate VLPbased vaccines against this important disease.

Insect cells infected with recombinant baculoviruses have also been used to produce infectious adeno-associated virus (AAV) type 2 vectors $^{74}$. Insect cells were coinfected with three recombinant baculoviruses, one expressing the AAV replication proteins, a second expressing the AAV structural proteins and a third expressing GFP under the control of a cytomegalovirus (CMV) promoter bounded by the AAV inverted terminal repeat sequences. The yield of functional genome-containing AAV particles per $S f 9$ cell produced in this system approached $5 \times 10^{4}$ demonstrating the system can produce large quantities of AAV vectors.

\section{Baculovirus display}

A variety of strategies have been developed for displaying heterologous peptides or proteins on the surface of baculovirus particles by fusing the peptide or protein to the baculovirus surface glycoprotein, gp $64^{75,76}$. In most instances the vector is designed so that baculovirus particles contain both wild-type gp64 and gp64 molecules containing the heterologous protein sequence. Baculoviruses displaying gp64fusion proteins have proven to be very effective immunogens. Since this approach was first used to raise monoclonal antibodies against the nuclear receptors LXR $\beta$ and $\mathrm{FXR}^{77}$, it has been used successfully to elicit antibody responses to a variety of displayed proteins. These include human peroxisome proliferator-activated receptor ${ }^{78}$, Plasmodium berghei circumsporozoite protein ${ }^{79}$, hemagglutinin protein of Rinderpest virus ${ }^{80}$, Theila parva sporozoite surface antigen $\mathrm{p} 67^{81}$ and foot-andmouth disease virus proteins ${ }^{82}$. Baculovirus display strategies have also been used for modification of the viral surface to influence baculovirus-mediated transduction of mammalian cells. These studies will be discussed further below. In addition to gp64 fusions, GFP was recently fused to the baculovirus vp39 capsid protein ${ }^{83}$. Capsid modifications may allow novel approaches for enhancing baculovirus mediated gene delivery into mammalian cells.
It has also been shown that membrane proteins produced in infected insect cells can be incorporated into baculovirus particles in a functional form. This was first observed for the $\beta$-2-adrenergic receptor, which was recovered in a functional form complexed with heterotrimeric $\mathrm{G}$ proteins ${ }^{84}$ and more recently for the human leukotriene $\mathrm{B} 4$ receptor $^{85}$. This approach has been used successfully to produce a functional $\gamma$-secretase complex on the surface of baculovirus particles ${ }^{86}$. Coinfection of $S f 9$ cells with viruses expressing the four putative $\gamma$-secretase components resulted in the production of virus particles with $\gamma$-secretase activity that was concentrated $\sim 2.5$-fold higher in the budded virus particles as compared to $S f 9$ cell membranes. These studies show that baculovirus particles can provide a unique scaffold for the assembly and enrichment of functional membrane bound protein complexes.

\section{Recombinant protein production in insect larvae}

The use of baculovirus-infected insect cell larvae as hosts for protein production was first described for the production of $\alpha$-interferon in 1985 (ref. 87). Since that time larvae have been used successfully to produce a variety of recombinant proteins ${ }^{88-92}$. This approach has been more widely adopted in Asian countries, including China, Japan and India, where silkworms are abundantly available and more laboratories have experience in growing and maintaining larvae. In most studies the expression vectors were based on Bombyx mori nucleopolyhedrosis virus (BmNPV), which infects the silkworm Bombyx mori. However, there is at least some industrial interest in the larvae of the cabbage looper moth, Trichoplusia ni, as host for recombinant protein production by Autographa californica nuclear polyhedrosis virus (AcMNPV)based recombinant baculovirus vectors in the United States, as well. Protein expression levels in baculovirus-infected larvae can be very high, reducing costs for large-scale production. Nevertheless, due to a general unfamiliarity with larval systems and ready access to cell culture facilities, this approach has not gained widespread popularity in most molecular biology laboratories in North America and Europe.

\section{Baculovirus-mediated gene delivery in mammalian cells}

The successful use of recombinant baculoviruses to direct gene expression in mammalian cells was first reported ten years ago ${ }^{93,94}$. Since we reviewed this subject ${ }^{95}$ there has been a remarkable increase in published reports of the use of this system. A number of publications have focused on improvements and demonstration of new cell types susceptible to baculovirus transduction, but most have described applications of this technology in areas such as genomics, pharmaceutical screening assays and in vivo applications such as gene therapy. In this section, we discuss advances that have been reported in the past few years.

Host cells and transduction parameters. The use of recombinant baculoviruses containing mammalian cell-active expression cassettes, commonly referred to as BacMam viruses, for gene delivery to mammalian cells was first demonstrated in cells of liver origin ${ }^{93,94}$. Subsequently, a number of labs reported gene delivery to a broad range of nonhepatic cell lines and primary cells ${ }^{96-98}$. Our 2002 review $^{95}$ contains a table of reported susceptible cells; however, there have been many recent additions. Primary rat chondrocytes are efficiently transduced by baculovirus ${ }^{99}$ and the transduced cells retain their differentiated state. Mouse primary kidney cells can express genes delivered by baculovirus for up to $20 \mathrm{~d}^{100}$. Hepatic stellate cells from rat and human are transduced at greater than $90 \%$ efficiency when the cells are activated by culturing on plastic surfaces, although transduction of fresh cultures is quite low $(<20 \%)^{101}$. Human osteosarcoma cell lines have been shown to be extremely good hosts for BacMammediated gene delivery ${ }^{102-104}$ surpassing even hepatoma cell lines in 
the observed level of gene expression. The efficient transduction of human mesenchymal stem cells has recently been demonstrated ${ }^{105}$. Though not mammalian, a number of fish cell lines also can be transduced at a low level that is improved by the addition of butyrate ${ }^{106}$. Furthermore, virus can be injected into the embryos of zebrafish to direct gene expression in vivo ${ }^{107}$.

One major advantage of the BacMam system is that gene delivery can be accomplished in many different cell types by simply adding a viral inoculum (Fig. 3). However, certain cell types that are not efficiently transduced can no doubt benefit by optimization of transduction conditions. Several parameters have been explored to improve transduction of HeLa cells ${ }^{108}$. Dilution of the virus inoculum with phosphate buffered saline rather than mammalian cell growth media as well as prolonged incubation of cells with the virus at low temperature $\left(25^{\circ} \mathrm{C}\right.$ rather than $\left.37^{\circ} \mathrm{C}\right)$ led to increases in gene expression. The addition of virus to HeLa cells in mid-log phase, followed by further additions of virus after several days allowed recombinant gene expression to be prolonged for over $10 \mathrm{~d}^{109}$. The addition of butyrate, a nonspecific inhibitor of histone deacetylase, has been shown to increase BacMam-mediated gene expression in a number of cell types ${ }^{98,110}$. Butyrate addition and incubation of cells at lower temperatures $\left(34^{\circ} \mathrm{C}\right.$ rather than $37^{\circ} \mathrm{C}$ ) were used to increase protein yields in transduced Chinese hamster ovary $(\mathrm{CHO})$ cells grown in suspension culture ${ }^{110}$. These investigators employed a modified $\mathrm{CHO}$ cell line that expresses the adenovirus E1A gene to further activate the CMV immediate early promoter and found that yields of micrograms of protein per $\mathrm{ml}$ of culture could be achieved. Enhancement of expression in transfected cells was observed using plasmids containing either the CMV or Drosophila melanogaster hsp70 gene promoter together with the baculoviral $h r l$ sequence ${ }^{111}$. A modest two- to threefold improvement in expression was observed in baculovirus transduction of mammalian cells when the $h r l$ sequence was added to the $h s p 70$ promoter. A comparison of BacMam gene delivery efficiency using four different promoters and four cell lines, in the presence and absence of the histone deacetylase inhibitor trichostatin A, demonstrated the cell line-dependent nature of the transduction process ${ }^{112}$.

Baculovirus entry. The entry mechanism and fate of baculovirus in mammalian cells are not well understood ${ }^{95}$, although some insights have been gained in recent years. When rat hepatocytes were cultured on collagen in medium containing DMSO to produce tight islands of cells, only cells on the periphery were transduced with baculovirus ${ }^{113}$. EGTA treatment disrupts the tight junctions, causes internalization of tight junction proteins, and allows the virus to transduce internal cells in these islands ${ }^{114}$. These findings imply that baculoviruses access the hepatocytes through the basolateral surface, which has implications for in vivo gene delivery to polarized epithelia. The fate of the virus after cell entry has been probed with a novel capsid protein-GFP fusion virus ${ }^{83}$. In cells that are transduced efficiently, the labeled viral nucleocapsid can be visualized in the nucleus by $4 \mathrm{~h}$ after transduction. However, in cells that are not transduced, the capsids do not travel to the nucleus suggesting that in certain cell types a defect in nuclear transport may block efficient baculovirus-mediated gene delivery.

The ability of the baculovirus envelope protein gp64 to accept fusions of other proteins and display them on the virion surface, as discussed above, has been exploited in attempts to target BacMam transduction of particular cells ${ }^{76}$. In general these attempts have led to increased binding of virus to cells, but no increased gene expression as compared to unmodified virions. However, a virus containing a fusion of avidin to gp64 has been shown to provide a higher transduction frequency in some cells ${ }^{115}$. A combination approach to improved transduction is

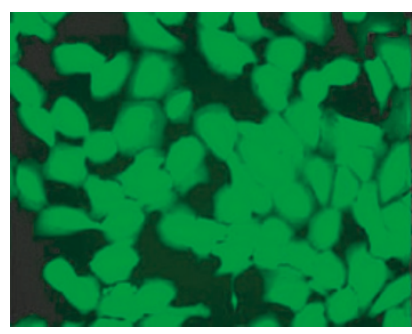

$\mathrm{U}-2$ OS

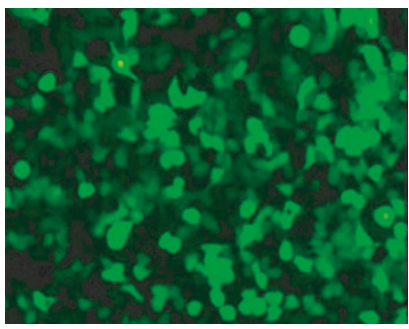

HEK 293

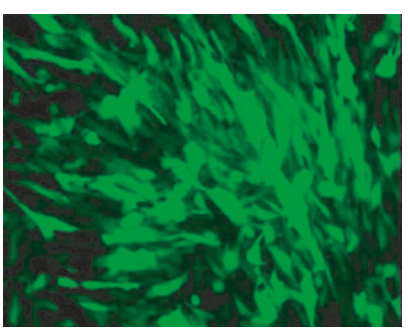

BHK

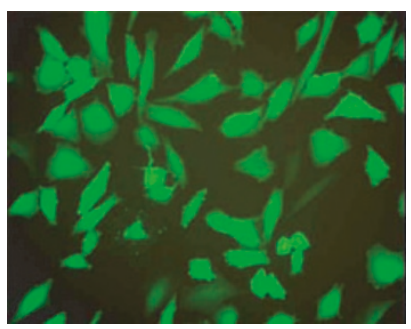

Saos-2
Figure 3 Photomicrograph of mammalian cells transduced with a BacMam virus expressing GFP. Virus as described in Condreay et al. ${ }^{98}$. The cells were transduced with 100 plaque-forming units of virus per cell and photographed 24 hrs after virus addition. U-2 OS, human osteosarcoma, BHK, baby hamster kidney, HEK 293, human embryonic kidney and Saos-2, human osteosarcoma cells. Transduction frequency of these cell types is routinely greater than $90 \%$ as measured by the number of fluorescent green cells.

suggested by these viral protein fusion technologies ${ }^{76}$. Fusions to the viral surface protein can be used to target virus to certain cell types, whereas fusion of nuclear targeting sequences to the capsid protein might facilitate nuclear delivery of the viral DNA.

In vivo gene delivery. BacMam viruses would appear to be ideal vectors for in vivo applications because they cannot replicate in mammalian cells, but can efficiently deliver genes into many cells types. Unfortunately, theory runs headlong into the reality that baculoviruses are rapidly inactivated by human serum complement ${ }^{116}$. A number of methods have been demonstrated to overcome this limitation and allow systemic delivery of virus in vivo ${ }^{117}$. After it was observed that a soluble form of complement receptor type 1 (sCR1) would protect baculovirus from complement inactivation in vitro ${ }^{118}, \mathrm{sCR} 1$ and virus were injected into the portal vein of mice, resulting in transgene expression in the livers of treated animals ${ }^{119}$. In these studies significant toxicity associated with viral delivery was attributed to the possible induction of inflammatory cytokines produced in response to the virus. It has been suggested that baculovirus particles pseudotyped with the vesicular stomatitis virus (VSV)-G protein are more resistant to complement than unmodified virus ${ }^{120}$. Viruses containing VSV-G were, in fact, more resistant to inactivation by human, rabbit, guinea pig, hamster and mouse serum, but not rat ${ }^{121}$. This modified virus could be used for in vivo gene transfer to cells in the cerebrum and testis, though no comparisons were made with the unmodified virus to assess improvements in efficiency. Another method used to accomplish in vivo gene delivery by baculovirus is to evade the complement system by careful choice of the route of administration ${ }^{117}$. Stereotaxic injection of virus into the striatum and vitreous body of rats resulted in transgene expression in neuronal cells distant from the injection site demonstrating that the virus can be transported through the axons of neurons ${ }^{122}$. A comparison of transgene delivery 


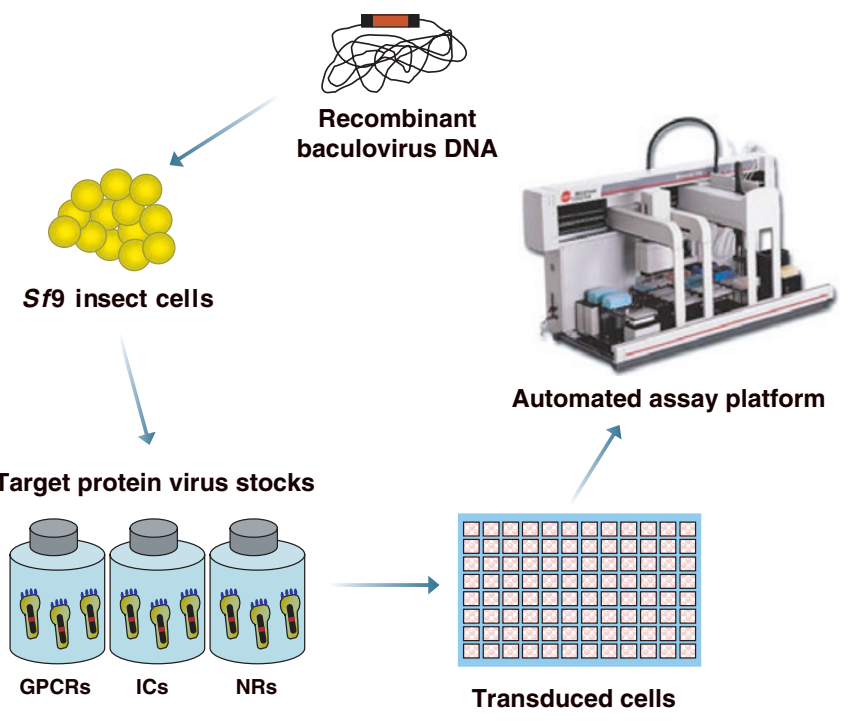

Figure4 Production of BacMam viruses expressing $\mathrm{G}$ protein-coupled receptor (GPCR), ion channel (IC) and nuclear receptor (NR) target proteins and transduction of mammalian cells in microtiter plate-based highthroughput assay formats. The system allows for a high level of flexibility in terms of target proteins, cell types and assay formats.

by baculovirus and adenovirus after injection into rat brain revealed that BacMam vectors targeted the choroid plexus, while adenovirus delivered genes to glial cells ${ }^{123}$.

\section{Baculoviruses as immunizing reagents}

The use of baculoviruses to express antigens under the control of mammalian cell-active promoters and elicit immune responses in vivo was first demonstrated by Aoki and colleagues ${ }^{124}$. Intramuscular inoculation with virus that expressed the pseudorabies gB protein elicited an anti-gB antibody response in mice. Similarly, antigen-specific immune responses were elicited with viruses expressing the HCV E2 protein and carcinoembryonic antigen as immunogens ${ }^{125}$. A VSV-G pseudotyped virus gave a similar response with tenfold less virus. A baculovirus that expresses the influenza hemagglutinin protein elicited an immune response when delivered by intramuscular or interperitoneal injection ${ }^{126}$. When either the hemagglutinin virus or wild-type virus were delivered intranasally, immunity was induced in the animals that protected them from a lethal challenge of influenza virus. This phenomenon was reported previously ${ }^{127}$. Treatment with baculovirus preparations could induce interferon production in cultured cells thus protecting the cells from infection by VSV. Similarly, mice injected with baculovirus became protected from a lethal challenge with encephalomyocarditis virus.

Gene function studies. The broad range of host cells susceptible to BacMam transduction, including primary cell cultures ${ }^{95}$, make the BacMam approach an excellent choice for in vitro studies of gene function. Baculovirus gene delivery has been used to deliver herpes virus genes to cells to elucidate their function ${ }^{128,129}$. Gene products can be studied in isolation from the parental herpes virus to determine their contribution to processes that happen during a normal infection, such as the mechanism behind the herpes virus $U_{S} 3$-mediated block of apoptosis ${ }^{130}$. Baculoviruses delivering truncated forms ${ }^{131}$ or mutant forms ${ }^{132}$ of glycoprotein $\mathrm{D}$ have been used to complement glycoprotein D-defective herpes viruses and dissect domains of the glycoprotein D protein that are involved in different events in infection. Delivery of the CMV IE2 gene with a recombinant baculovirus facilitated studies on the role of this gene product in regulating viral gene expression ${ }^{133}$ and cellular gene expression that might be involved in viral pathogenicity ${ }^{134}$.

The in vitro study of hepatitis B virus (HBV) and $\mathrm{HCV}$ virus is hindered by the lack of cell culture systems to propagate these viruses. For HBV this problem was overcome by using a baculovirus to deliver the HBV genome to hepatoma cells and launch a productive HBV infection ${ }^{135}$. This approach has been used to investigate the action of anti-viral compounds on HBV infection ${ }^{136}$, probe the characteristics of drug-resistant mutants of $\mathrm{HBV}^{137}$ and characterize the reinitiation of viral infection after anti-viral treatment ${ }^{138}$. Analogous to HBV delivery, baculoviruses have been used to deliver a cDNA copy of the HCV genome to cells ${ }^{139}$. Although HCV RNA replication or virions were not detected, the HCV polyprotein was produced and properly processed. McCormick and colleagues have extended this observation and produced baculoviruses with inducible expression of HCV genomes to study viral replication ${ }^{140,141}$.

The discovery that BacMam viruses could be used to deliver genes to specific regions of the developing zebrafish has made it possible to elucidate gene function by observing the effects of misexpressing proteins in the whole animal ${ }^{107,142}$. When virus is injected into early-stage embryos, transgene expression is disseminated, but when it is injected at later stages, expression is limited to the injection site ${ }^{107}$.

RNA interference has become a widely used tool to study the effects of reducing the expression of targeted gene products. A prerequisite for successful RNA interference is efficient delivery of the interfering RNA. Recently a baculovirus engineered to express short hairpin RNA from the U6 promoter has been used to reduce expression of lamin A/C RNA and protein in human cell lines (Saos2, HepG2 and Huh7) and primary hepatic stellate cells ${ }^{143}$. These results indicate that baculoviruses provide another useful delivery approach for delivering interfering RNAs.

Cell-based assays. The development of cell-based assays for highthroughput screening of chemical libraries has traditionally involved using stable cell lines producing the target protein(s). In some instances developing and culturing these cell lines is problematic due to the deleterious effects of the target gene product(s). There are a number of recent reports illustrating how BacMam-mediated transient expression can be used to overcome this difficulty for cell-based assay development in automated facilities. Assays to screen for modulators of protein activity have been described for ion channels ${ }^{144}$, nuclear receptors ${ }^{104,145}$ and GPCRs ${ }^{146,147}$. We have previously mentioned viral replication assays suitable for this purpose ${ }^{136}$. Jenkinson et al. ${ }^{148}$. have described a novel assay using BacMam-delivered human immunodeficiency virus gene products to mimic the receptor-mediated membrane fusion event required for viral infection that is amenable to a high-throughput screening format. Another interesting application involves using fixed monolayers of BacMam-GCPR-transduced cells to screen for antibodies destined for immunohistochemistry studies ${ }^{149}$. The versatility of the BacMam system for pharmaceutical screening assays is illustrated in Figure 4. Large libraries of these viral reagents can be easily generated making gene delivery to the proper cell type for assay a simple liquid delivery step that can be performed on automated platforms.

\section{Conclusions and future developments}

The baculovirus-insect cell expression system has proven to be an extremely valuable tool for recombinant protein production. Ongoing improvements in vector design and simplification of recombinant virus isolation techniques, combined with the relative ease of small 
and large scale culture of insect cells, have resulted in widespread use of this system. Many laboratories are beginning to automate the production of large numbers of viruses and protein production schemes using advanced cloning methods, robotic liquid handling and protein purification instruments. Genetic transformation has been used to create transgenic insect cells with humanized protein glycosylation pathways, which can be used as improved hosts for baculovirus expression vectors, enabling the production of more authentic mammalian $N$-glycoproteins. In the future, we can expect to see analogous transgenic insect cell lines engineered in other ways to improve their protein processing capabilities and enhance their ability to produce properly folded and modified recombinant proteins. In addition, the application of the baculovirus-insect cell system for the production of VLPs and functional multi-subunit complexes will continue to provide reagents that are difficult to produce in any other way.

Perhaps the most unexpected development discussed in this review is the increasing use of recombinant baculoviruses as gene delivery vectors for mammalian cells. We believe this application will ultimately become as commonplace as the use of recombinant baculoviruses for recombinant protein production. This relatively new gene delivery approach offers a number of advantages including: the inability of the virus to replicate in mammalian cells, the absence or virtual absence of cytotoxicity, technical simplicity and a superior biosafety profile $\mathrm{e}^{150}$ as compared to mammalian cell-derived viral vectors. The characterization of this gene delivery application is in its infancy and it will be especially important to gain additional understanding of the mechanisms involved in viral transduction. An increased knowledge of the baculovirus-cell interactions that result in efficient gene transfer will direct future efforts to enhance vector design and/or engineer cells to increase transduction efficiency. Finally, the application of baculodisplay technologies may also prove useful to improve and extend the variety of host cells that can be efficiently transduced.

\section{ACKNOWLEDGMENTS}

T.A.K. and J.P.C. thank John Gray, Mike Romanos and John Reardon at

GlaxoSmithKline for their continued support and encouragement of the development and application of baculovirus technology. D.L.J. thanks the National Institutes of Health (GM49734) and the National Science Foundation (BES9814157 and BES9818001) for generously supporting baculovirus-insect cell work in his laboratory. The authors appreciate the assistance of James Frye in preparing the figures.

\section{COMPETING INTERESTS STATEMENT}

The authors declare competing financial interests (see the Nature Biotechnology website for details).

Published online at http://www.nature.com/naturebiotechnology/

1. Luckow, V.L. \& Summers, M.D. Trends in the development of baculovirus expression vectors. Bio/Technology 6, 47-55 (1988)

2. O'Reilly, D.R., Miller, L.K. \& Luckow, V.A. Baculovirus Expression Vectors: A Laboratory Manual (W.H. Freeman, New York, 1992).

3. Miller, L.K. (ed.). The Baculoviruses (Plenum Press, New York, 1997).

4. Possee, R.D. Baculoviruses as expression vectors. Curr. Opin. Biotechnol. 8, 569572 (1997).

5. Kost, T.A. \& Condreay, J.P. Recombinant baculoviruses as expression vectors for insect and mammalian cells. Curr. Opin. Biotechnol. 10, 428-433 (1999).

6. Freisen, D.P. \& Miller, L.K. Insect viruses. in Fields' Virology, edn. 4, (eds. Knipe, D.M. et al.) 1871-1940 (Lippincott Williams and Wilkins, Philadelphia, 2001).

7. Kitts, P.A., Ayres, M.D. \& Possee, R.D. Linearization of baculovirus DNA enhances the recovery of recombinant virus expression vectors. Nucleic Acids Res. 18, 5667$5672(1990)$

8. Kitts, P.A. \& Possee, R.D. A method for producing recombinant baculovirus expression vectors at high frequency. Biotechniques 14, 810-817 (1993).

9. Luckow, V.A., Lee, S.C., Barry, G.F. \& Olins, P.O. Efficient generation of infectious recombinant baculoviruses by site-specific transposon-mediated insertion of foreign genes into a baculovirus genome propagated in Escherichia coli. J . Virol. 67, 4566-4579 (1993).

10. Airenne, K.J., Peltomaa, E., Hytönen, V.P., Laitinen, O.H. \& Ylä-Herttuala, S. Improved generation of recombinant baculovirus genomes in Escherichia coli. Nucleic Acids Res. 31, e101 (2003).
11. Laitinen et al. A multipurpose vector system for the screening of libraries in bacteria, insect and mammalian cells and expression in vivo. Nucleic Acids Res. 33, e42 (2005).

12. Pijlman, G.P., van den Born, E., Martens, D.E. \& Vlak, J.M. Autographa californica baculoviruses with large genomic deletions are rapidly generated in infected insect cells. Virology 283, 132-138 (2001).

13. Pijlman, G.P., van Schijndel, J.E. \& Vlak, J.M. Spontaneous excision of BAC vector sequences from bacmid-derived baculovirus expression vectors upon passage in insect cells. J. Gen. Virol. 84, 2669-2678 (2003).

14. Zhao, Y., Chapman, D.A. \& J ones, I.M. Improving baculovirus recombination. Nucleic Acids Res. 31, e6 (2003).

15. Chambers, S.P., Austen, D.A., Fulghum, J.R. \& Kim, W.M. High-throughput screening for soluble recombinant expressed kinases in Escherichia coli and insect cells. Protein Expr. Purif. 36, 40-47 (2004).

16. Page, R. et al. Scalable high-throughput micro-expression device for recombinant proteins. Biotechniques 37, 364-368 (2004).

17. Bahia, D., Cheung, R., Buchs, M., Geisse, S. \& Hunt, I. Optimization of insect cell growth in deep-well blocks: development of a high-throughput insect cell expression screen. Protein Expr. Purif. 39, 61-70 (2005).

18. Kitts, P.A. \& Green, G. An immunological assay for determination of baculovirus titers in 48 hours. Anal. Biochem. 268, 173-178 (1999).

19. Kwon, M.S., Dojima, T., Toriyama, M. \& Park, E.Y. Development of an antibodybased assay for determination of baculovirus titers in 10 hours. Biotechnol. Prog 18, 647-651 (2002).

20. Mulvania, T., Hayes, B. \& Hedin, D. A flow cytometric assay for rapid, accurate determination of baculovirus titers. BioProcessing J. 3, 47-53 (2004).

21. Shen, C.F., Meghrous, J. \& Kamen, A. Quantitation of baculovirus particles by flow cytometry. J. Virol. Methods 105, 321-330 (2002).

22. LO, H.R. \& Chao, Y.C. Rapid titer determination of baculovirus by quantitative realtime polymerase chain reaction. Biotechnol. Prog. 20, 354-360 (2004).

23. J arvis, D.L. Baculovirus expression vectors. in The Baculoviruses. (ed. Miller, L.K.) 389-431 (Plenum Press, New York, 1997).

24. Marz, L., Altmann, F., Staudacher, E. \& Kubelka, V. Protein glycosylation in insects. in Glycoproteins, vol. 29a. (eds. Montreuil, J., Vliegenthart, J .F.G. \& Schachter, H.) 543-563 (Elsevier, Amsterdam, 1995).

25. Marchal, I., Jarvis, D.L., Cacan, R. \& Verbert, A. Glycoproteins from insect cells: sialylated or not? Biol. Chem. 382, 151-159 (2001).

26. Altmann, F., Schwihla, H., Staudacher, E., Glossl, J. \& Marz, L. Insect cells contain an unusual, membrane-bound $\beta-\mathrm{N}$-acetylglucosaminidase probably involved in the processing of protein N-glycans. J. Biol. Chem. 270, 17344-17349 (1995).

27. J arvis, D.L., Kawar, Z.S. \& Hollister, J.R. Engineering N-glycosylation pathways in the baculovirus-insect cell system. Curr. Opin. Biotechnol. 9, 528-533 (1998).

28. Jarvis, D.L. Humanizing recombinant glycoprotein production in the baculovirusinsect cell expression system. Virology 310, 1-7 (2003).

29. Tomiya, N., Betenbaugh, M.J . \& Lee, Y.C. Humanization of lepidopteran insect-cellproduced glycoproteins. Acc. Chem. Res. 36, 613-620 (2003).

30. Tomiya, N., Narang, S., Lee, Y.C. \& Betenbaugh, M.J . Comparing N-glycan processing in mammalian cell lines to native and engineered lepidopteran insect cell lines. Glycoconj. J. 21, 343-360 (2004).

31. Betenbaugh, M.J., Tomiya, N., Narang, S., Hsu, J.T. \& Lee, Y.C. Biosynthesis of human-type N-glycans in heterologous systems. Curr. Opin. Struct. Biol. 14,601606 (2004).

32. Jarvis, D.L., Fleming, J.A., Kovacs, G.R., Summers, M.D. \& Guarino, L.A. Use of early baculovirus promoters for continuous expression and efficient processing of foreign gene products in stably transformed lepidopteran cells. Bio/Technology $\mathbf{8}$ 950-955 (1990)

33. Jarvis, D.L., Weinkauf, C. \& Guarino, L.A. Immediate early baculovirus vectors for foreign gene expression in transformed or infected insect cells. Protein Expr. Purif. 8, 191-203 (1996).

34. Hollister, J.R., Shaper, J.H. \& J arvis, D.L. Stable expression of mammalian beta 1,4-galactosyltransferase extends the $\mathrm{N}$-glycosylation pathway in insect cells. Glycobiology 8, 473-480 (1998).

35. Hollister, J.\&J arvis, D.L. Engineering lepidopteran insect cells for sialoglycoprotein production by genetic transformation with mammalian $\beta 1,4$-galactosyltransferase and a2,6-sialyltransferase genes. Glycobiology 11, 1-9 (2001).

36. Hooker, A.D. et al. Constraints on the transport and glycosylation of recombinant IFN-gamma in chinese hamster ovary and insect cells. Biotech. Bioeng. 63, 559572 (1999).

37. Tomiya, N., Ailor, E., Lawrence, S.M., Betenbaugh, M.J . \& Lee, Y.C. Determination of nucleotides and sugar nucleotides involved in protein glycosylation by high-performance anion-exchange chromatography: sugar nucleotide contents in cultured insect cells and mammalian cells. Anal. Biochem. 293, 129-137 (2001).

38. Hollister, J., Conradt, H.O. \& J arvis, D.L. Evidence for a sialic acid salvaging pathway in lepidopteran insect cell lines. Glycobiology 13, 487-495 (2003).

39. Hollister, J., Grabenhorst, E., Nimtz, M., Conradt, H.O. \& J arvis, D.L. Engineering the protein $\mathrm{N}$-glycosylation pathway in insect cells for production of biantennary, complex N-glycans. Biochemistry 41, 15093-15104 (2002).

40. Aumiller, J.J., Hollister, J.R. \& J arvis, D.L. A transgenic lepidopteran insect cell line engineered to produce CMP-sialic acid and sialoglycoproteins. Glycobiology 13, 497-507 (2003).

41. Ailor, E. \& Betenbaugh, M.J. Modifying secretion and post-translational processing in insect cells. Curr. Opin. Biotechnol. 10, 142-145 (1999).

42. Higgins, M.K., Demir, M. \& Tate, C.G. Calnexin co-expression and the use of weaker promoters increase expression of correctly assembled shaker potassium channel in 
insect cells. Biochim. Biophys. Acta 1610, 124-132 (2003)

43. Zhang, L., Wu, G., Tate, C.G., Lookene, A. \& Olivecrona, G. Calreticulin promotes folding/dimerization of human lipoprotein lipase expressed in insect cells. (Sf21). J. Biol. Chem. 278, 29344-29351 (2003).

44. Fourneau, J.M., Cohen, H. \& van Endert, P.M. A chaperone assisted high yield system for the production of HLA-DR4 tetramers in insect cells. J. Immunol. Methods 285, 253-264 (2004).

45. Yokoyama, N. et al. Co-expression of human chaperones Hsp70 and Hsdj or Hsp4O cofactor increases solubility of overexpressed target proteins in insect cells. Biochim. Biophys. Acta 1493, 119-124 (2000).

46. Martinez-Torrecuadrada, J.L. et al. An efficient expression system for the production of functionally active human LKB1. J. Biotechnol. 115, 23-34 (2005).

47. Venkaiah, B., Viswanathan, P., Habib, S. \& Hasnain, S.E. An additional copy of the homologous region ( $\mathrm{hr} 1$ ) sequence in the Autographa californica multinucleocapsid polyhedrosis virus genome promotes hyperexpression of foreign genes. Biochemistry 43, 8143-8151 (2004)

48. Chen, Y. et al. A constitutive super-enhancer: homologous region 3 of bombyx mori nucleopolyhedrosis virus. Biochem. Biophys. Res. Commun. 318, 1039-1044 (2004)

49. Sano, K., Maeda, K., Oki, M., \& Maeda, Y. Enhancement of protein expression in insect cells by a lobster tropomysin cDNA leader sequence. FEBS Lett. 532, 143-146 (2002).

50. Ho, Y., Lo, H.R., Lee, T.C., Wu, C.P.Y. \& Chao, Y.C. Enhancement of correct protein folding in vivo by a non-lytic baculovirus. Biochem. J. 382, 695-702 (2004).

51. Kaba, S.A., Salcedo, A.M., Wafula, P.O., Vlak, J.M. \& van Oers, M.M. Development of a chitinase and $v$-cathepsin negative bacmid for improved integrity of secreted recombinant proteins. J. Virol. Methods 122, 113-118 (2004).

52. Sarramegna, V., Talmont, P., Demange, P. \& Milon, A. Heterologous expression of G-protein-coupled receptors: comparison of expression systems from the standpoint of large-scale production and purification. Cell. Mol. Life Sci. 60, 1529-1546 (2003).

53. Massotte, D. Protein-coupled receptor overexpression with the baculovirus-insect cell expression system: a tool for structural and functional studies. Biochim. Biophys. Acta 1610, 77-89 (2003)

54. Gazi, L., Nickolls, S.A. \& Strange, P.G. Functional coupling of the human dopamine $\mathrm{D} 2$ receptor with $\mathrm{G} \alpha_{\mathrm{i} 1}, \mathrm{G \alpha}_{\mathrm{i} 2}, \mathrm{G} \alpha_{\mathrm{i3}}$ and $\mathrm{G}_{\alpha 0} \mathrm{G}$ proteins: evidence for agonist regulation of $\mathrm{G}$ protein selectivity. Br. J. Pharmacol. 138, 775-786 (2003).

55. Hood, S.R., Shah, G. \& J ones, P. Expression of cytochrome P450 in a baculovirus system. Methods Mol. Biol. 107, 203-218 (1998).

56. Rushmore, T.H., Reider, P.J ., Slaughter, D., Assang, C. \& Shou, M. Bioreactor systems in drug metabolism: synthesis of cytochrome P450-generated metabolites. Metab. Eng. 2, 115-125 (2000).

57. Sanchez, R.I. et al. Cytochrome p450 $3 a 4$ is the major enzyme involved in the metabolism of the substance $P$ receptor antagonist aprepitant. Drug Metab. Dispos. 32, 1287-1292 (2004).

58. Ikonomou, L., Schneider, Y.J . \& Agathos, S.N. Insect cell culture for industrial production of recombinant proteins. Appl. Microbiol. Biotechnol. 62, 1-20 (2003).

59. Maranga, L., Cruz, P.E., Aunins, J.A. \& Carrondo, M.J.T. Production of core and viruslike-particles with baculovirus infected insect cells. Adv. Biochem. Eng. Biotechnol. 74, 183-206 (2002)

60. Noad, R. \& Roy, P. Virus-like particles as immunogens. Trends Microbiol. 11, 438 444 (2003).

61. Garcea, R.L. \& Gissman, L. Virus-like particles as vaccines and vessels for the delivery of small molecules. Curr. Opin. Biotechnol. 15, 513-517 (2004)

62. Petry, H., Goldmann, C., Ast, O. \& Luke, W. The use of virus-like particles for gene transfer. Curr. Opin. Mol. Ther. 5, 524-528 (2003).

63. Berger, I., Fitzgerald, D.J . \& Richmond, T.J. Baculovirus expression system for heterologous multiprotein complexes. Nat. Biotechnol. 22, 1583-1587 (2004).

64. Fausch, S.C., DaSilva, D.M., Eiben, G.L., LePoole, I.C. \& Kast, W.M. HPV protein/ peptide vaccines: from animal models to clinical trials. Front. Biosci. 8, s81-s91 (2003)

65. Stanley, M.A. Progress in prophylactic and therapeutic vaccines for human papilloma infection. Expert Rev. Vaccines 2, 381-389 (2003).

66. Harper, D.M. et al. Efficacy of a bivalent $L 1$ virus-like particle vaccine in prevention of infection with human papilloma types 16 and 18 in young women: a randomised controlled trial. Lancet 364, 1757-1765 (2004).

67. Baumert, T.F., Ito, S., Wong, D.T. \& Liang, T.J . Hepatitis C virus structural proteins assemble into virus-like particles in insect cells. J. Virol. 72 3827-3836 (1998).

68. Xiang, J. et al. Recombinant hepatitis $C$ virus-like particles expressed by baculovirus: utility in cell-binding and antibody detection assay. J. Med. Virol. 68, 537-543 (2002).

69. Choi, S.H., Kim, S.Y., Park, K.J., Kim, Y.J . \& Hwang, S.B. Hepatitis C virus core protein is efficiently released into the culture medium in insect cells. J. Biochem. Mol. Biol. 37, 735-740 (2004).

70. Girard, C. et al. Effect of the $5^{\prime}$ non-translated region on self assembly of hepatitis $C$ virus genotype 1 a structural proteins produced in insect cells. J. Gen. Virol. 85, 3659-3670 (2004).

71. J eong, S.H. et al. Immunization with hepatitis $\mathrm{C}$ virus-like particles induces humoral and cellular immune responses in nonhuman primates. J. Virol. 78, 6995-7003 (2004).

72. Ho, Y., Lin, P.H., Liu, C.Y.Y., Lee, S.P. \& Chao, Y.C. Assembly of human severe acute respiratory syndrome coronavirus-like particles. Biochem. Biophys. Res. Commun. 318, 833-838 (2004).

73. Mortola, E. \& Roy, P. Efficient assembly and release of SARS coronavirus-like particles by a heterologous expression system. FEBS Lett. 576, 174-178 (2004)

74. Urabe, M., Ding, C. \& Kotin, R.M. Insect cells as a factory to produce adeno-associated virus type 2 vectors. Hum. Gene Ther. 13, 1935-1943 (2002).

75. Boublik, Y., Di Bonito, P. \& J ones, I.M. Eukaryotic virus display: engineering the major surface glycoprotein of the Autographa californica nuclear polyhedrosis virus (ACNPV) for the presentation of foreign proteins on the virus surface. Bio/Technology 13, 1079-1084 (1995)

76. Oker-Blom, C., Airenne, K.J \& \& Grabherr, R. Baculovirus display strategies: emerging tools for eukaryotic libraries and gene delivery. Brief. Funct. Genomic. Proteomic. 2. 244-253 (2003).

77. Lindley, K. et al. Production of monoclonal antibodies using recombinant baculovirus displaying gp64-fusion proteins. J. Immunol. Methods 234, 123-135 (2000).

78. Tanaka, T. et al. The generation of monoclonal antibodies against human peroxisome proliferator-activated receptors (PPARs). J. Atheroscler. Thromb. 9, 233-242 (2002).

79. Yoshida, S. et al. Baculovirus virions displaying Plasmodium berghei circumsprozoite protein protect mice against malaria sporozoite infection. Virology 316, 161-170 (2003).

80. Rahman, M.M. Shaila, M.S. \& Gopinathan, K.P. Baculovirus display of fusion protein of Pestes des petits ruminants virus and hemagglutination protein of rinderpest virus and immunogenicity of the displayed proteins in mouse model. Virology 317 36-49 (2003).

81. Kaba, S.A., Nene, V., Musoke, A.J., Vlak, J.M. \& van Oers, M.M. Fusion to green fluorescent protein improves expression of Theileria parva sporozoite surface antigen p67 in insect cells. Parasitology 125, 497-505 (2002)

82. Tami, C. et al. Immunological properties of FMDV-gp64 fusion proteins expressed on SF9 cell and baculovirus surfaces. Vaccine 23, 840-845 (2004).

83. Kukkonen, S.P. et al. Baculovirus capsid display: a novel tool for transduction imaging $\mathrm{Mol}$. Ther 8, 853-862 (2003).

84. Loisel, T.P. et al. Recovery of homogeneous and functional beta 2-adrenergic receptors from extracellular baculovirus particles. Nat. Biotechnol. 15, 1300-1304 (1997).

85. Masuda, K. et al. A combinatorial G protein-coupled receptor reconstitution system on budded baculovirus. Evidence for Galpha and Galphao coupling to a human leukotriene B4 receptor. J. Biol. Chem. 278, 24552-24562 (2003).

86. Hayashi, I. et al. Selective reconstitution and the recovery of functional $\gamma$-secretase complex on budded baculovirus particles. J. Biol. Chem. 279, 38040-38046 (2004).

87. Maeda, S. et al. Production of human alpha-interferon in silkworm using a baculovirus vector. Nature 315, 592-594 (1985).

88. Wu, D. et al. Expression of biologically active recombinant equine interferon- $\gamma$ by two different expression systems using insect cells and silkworm larvae. Cytokine 20, 63-69 (2002)

89. Sehgal, D., Malik, P.S. \& J ameel, S. Purification and diagnostic utility of a recombinant hepatitis E virus capsid protein expressed in insect larvae. Protein Expr. Purif. 27, 27-34 (2003)

90. Muneta, Y. et al. Expression and one-step purification of bovine interleukin-21 (IL21 ) in silkworms using a hybrid baculovirus expression system. Biotechnol. Lett. 26, 1453-1458 (2004)

91. Nagaya, H. et al. Establishment of a large-scale purification procedure for purification of recombinant bovine interferon-tau produced by a silkworm-baculovirus gene expression system. I. Vet. Med. Sci. 66, 1395-1401 (2004).

92. Motohashi, T. Shimojima, T., Fukagawa, T., Maenaka, K. \& Park, E.Y. Efficient large-scale production of larvae and pupae of silkworm by Bombyx mori nuclea polyhedrosis virus bacmid system. Biochem. Biophys. Res. Commun. 326, 564-569 (2005)

93. Hofmann, C. et al. Efficient gene transfer into human hepatocytes by baculovirus vectors. Proc. Natl. Acad. Sci. USA 92, 10099-10103 (1995).

94. Boyce, F.M. \& Bucher, N.L. Baculovirus-mediated gene transfer into mammalian cells. Proc. Natl. Acad. Sci. USA 93, 2348-2352 (1996).

95. Kost, T.A. \& Condreay, J.P. Recombinant baculoviruses as mammalian cell genedelivery vectors. Trends Biotechnol. 20, 173-180 (2002).

96. Shoji, I. et al. Efficient gene transfer into various mammalian cells, including non hepatic cells, by baculovirus vectors. J. Gen. Virol. 78, 2657-2664 (1997).

97. Yap, C.C. et al. A hybrid baculovirus-T7 RNA polymerase system for recovery of an infectious virus from cDNA. Virology 231, 192-200 (1997)

98. Condreay, J .P., Witherspoon, S.M., Clay, W.C. \& Kost, T.A. Transient and stable gene expression in mammalian cells transduced with a recombinant baculovirus vector. Proc. Natl. Acad. Sci. USA 96, 127-132 (1999).

99. Ho, Y.C., Chen, H.C., Wang, K.C. \& Hu, Y.C. Highly efficient baculovirus-mediated gene transfer into rat chondrocytes. Biotechnol. Bioeng. 88, 643-651 (2004).

100. Liang, C.Y., Wang, H.Z., Li, T.X., Hu, Z.H. \& Chen, X.W. High efficiency gene transfer into mammalian kidney cells using baculovirus vectors. Arch. Virol. 149, 51-60 (2004)

101. Gao, R. et al. High efficiency gene transfer into cultured primary rat and human hepatic stellate cells using baculovirus vectors. Liver 22, 15-22 (2002).

102. Song, S.U. \& B oyce, F.M. Combination treatment for osteosarcoma with baculovira vector mediated gene therapy (p53) and chemotherapy (adriamycin). Exp. Mol. Med. 33, 46-53 (2001)

103. Song, S.U. et al. Effective transduction of osteogenic sarcoma cells by a baculovirus vector. J. Gen. Virol. 84, 697-703 (2003)

104. Clay, W.C. et al. Recombinant baculoviruses used to study estrogen receptor function in human osteosarcoma cells. Assay Drug Dev. Technol. 1, $801-810$ (2003).

105. Ho, Y.C., Chung, Y.C., Hwang, S.M., Wang, K.C. \& Hu, Y.C. Transgene expression 
and differentiation of baculovirus-transduced human mesenchymal stem cells. J. Gene Med.; published online 11 February 2005 (doi: 10.1002/jgm.729).

106. Leisy, D.J., Lewis, T.D., Leong, J.A. \& Rohrmann, G.F. Transduction of cultured fish cells with recombinant baculoviruses. J. Gen. Virol. 84, 1173-1178 (2003).

107. Wagle, M. \& J esuthasan, S. Baculovirus-mediated gene expression in zebrafish. Mar. Biotechnol. (NY) 5, 58-63 (2003)

108. Hsu, C.S., Ho, Y.C., Wang, K.C. \& Hu, Y.C. Investigation of optimal transduction conditions for baculovirus-mediated gene delivery into mammalian cells. Biotechnol. Bioeng. 88, 42-51 (2004).

109. Hu, Y.C., Tsai, C.T., Chang, Y.J . \& Huang, J.H. Enhancement and prolongation of baculovirus-mediated expression in mammalian cells: focuses on strategic infection and feeding. Biotechnol. Prog. 19, 373-379 (2003)

110. Ramos, L et al. Rapid expression of recombinant proteins in modified $\mathrm{CHO}$ cells using the baculovirus system. Cytotechnology 38, 37-41 (2002).

111. Viswanathan, P. et al. The homologous region sequence (hr1) of Autographa californica multinucleocapsid polyhedrosis virus can enhance transcription from nonbaculoviral promoters in mammalian cells. J. Biol. Chem. 278, 52564-52571 (2003).

112. Spenger, A., Ernst, W., Condreay, J.P., Kost, T.A. \& Grabherr, R. Influence of promoter choice and trichostatin A treatment on expression of baculovirus delivered genes in mammalian cells. Protein Expr. Purif. 38, 17-23 (2004).

113. Bilello, J.P., Delaney, W.E., Boyce, F.M. \& Isom, H.C. Transient disruption of intercellular junctions enables baculovirus entry into nondividing hepatocytes. J. Virol. 75, 9857-9871 (2001)

114. Bilello, J.P., Cable, E.E., Myers, R.L. \& Isom, H.C. Role of paracellular junction complexes in baculovirus-mediated gene transfer to nondividing rat hepatocytes. Gene Ther. 10, 733-749 (2003)

115. Räty, J.K. et al. Enhanced gene delivery by avidin-displaying baculovirus. Mol. Ther 9. 282-291 (2004).

116. Hofmann, C. \& Strauss, M. Baculovirus-mediated gene transfer in the presence of human serum or blood facilitated by inhibition of the complement system. Gene Ther. 5, 531-536 (1998).

117. Huser, A. \& Hofmann, C. Baculovirus vectors: novel mammalian cell gene-delivery vehicles and their applications. Am. J. Pharmacogenomics 3, 53-63 (2003).

118. Hofmann, C., Huser, A., Lehnert, W. \& Strauss, M. Protection of baculovirus-vectors against complement-mediated inactivation by recombinant soluble complement receptor type 1. Biol. Chem. 380, 393-395 (1999).

119. Hoare, J., Waddington, S., Thomas, H.C., Coutelle, C. \& McGarvey, M.J . Complement inhibition rescued mice allowing observation of transgene expression following intraportal delivery of baculovirus in mice. J. Gene Med. 7, 325-333 (2005).

120. Barsoum, J., Brown, R., McKee, M. \& Boyce, F.M. Efficient transduction of mammalian cells by a recombinant baculovirus having the vesicular stomatitis virus $G$ glycoprotein. Hum. Gene Ther. 8, 2011-2018 (1997).

121. Tani, H. et al. In vitro and in vivo gene delivery by recombinant baculoviruses. J. Virol. 77, 9799-9808 (2003)

122. Li, Y., Wang, X., Guo, H. \& Wang, S. Axonal transport of recombinant baculovirus vectors. Mol. Ther. 10, 1121-1129 (2004)

123. Lehtolainen, P., Tyynela, K., Kannasto, J., Airenne, K.J.\& Ylä-Herttuala, S. Baculoviruses exhibit restricted cell type specificity in rat brain: a comparison of baculovirus- and adenovirus-mediated intracerebral gene transfer in vivo. Gene Ther. 9, 1693-1699 (2002)

124. Aoki, H. et al. Induction of antibodies in mice by a recombinant baculovirus expressing pseudorabies virus glycoprotein B in mammalian cells. Vet. Microbiol. 68, 197207 (1999).

125. Facciabene, A., Aurisicchio, L. \& La Monica, N. Baculovirus vectors elicit antigenspecific immune responses in mice. J. Virol. 78, 8663-8672 (2004).

126. Abe, $T$. et al. Baculovirus induces an innate immune response and confers protection from lethal influenza virus infection in mice. J. Immunol. 171, 1133-1139 (2003).

127. Gronowski, A.M., Hilbert, D.M., Sheehan, K.C., Garotta, G. \& Schreiber, R.D. Baculovirus stimulates antiviral effects in mammalian cells. J. Virol. 73, 9944-9951 (1999).

128. Ye, G.J., Vaughan, K.T., Vallee, R.B. \& Roizman, B. The herpes simplex virus 1 $\mathrm{U}(\mathrm{L}) 34$ protein interacts with a cytoplasmic dynein intermediate chain and targets nuclear membrane. J. Virol. 74, 1355-1363 (2000).
129. Zhou, G., Galvan, V., Campadelli-Fiume, G. \& Roizman, B. Glycoprotein D or J delivered in trans blocks apoptosis in SK-N-SH cells induced by a herpes simplex virus 1 mutant lacking intact genes expressing both glycoproteins. J. Virol. 74 11782-11791 (2000)

130. Benetti, L. \& Roizman, B. Herpes simplex virus protein kinase US3 activates and functionally overlaps protein kinase A to block apoptosis. Proc. Natl. Acad. Sci. USA 101, 9411-9416 (2004).

131. Zhou, G. \& Roizman, B. Truncated forms of glycoprotein D of herpes simplex virus 1 capable of blocking apoptosis and of low-efficiency entry into cells form a heterodimer dependent on the presence of a cysteine located in the shared transmembrane domains. J. Virol. 76, 11469-11475 (2002).

132. Zhou, G., Avitabile, E., Campadelli-Fiume, G. \& Roizman, B. The domains of glycoprotein $D$ required to block apoptosis induced by herpes simplex virus 1 are largely distinct from those involved in cell-cell fusion and binding to nectin1. J. Virol. 77, 3759-3767 (2003).

133. Kronschnabl, M., Marschall, M. \& Stamminger, T. Efficient and tightly regulated expression systems for the human cytomegalovirus major transactivator protein IE2p86 in permissive cells. Virus Res. 83, 89-102 (2002).

134. Kronschnabl, M. \& Stamminger, T. Synergistic induction of intercellular adhesion molecule- 1 by the human cytomegalovirus transactivators IE2p86 and pp71 is mediated via an Sp1-binding site. J. Gen. Virol. 84, 61-73 (2003).

135. Delaney, W.E. \& Isom, H.C. Hepatitis B virus replication in human HepG2 cells mediated by hepatitis B virus recombinant baculovirus. Hepatology $\mathbf{2 8}, 1134-1146$ (1998).

136. Delaney, W.E., Miller, T.G. \& Isom, H.C. Use of the hepatitis B virus recombinant baculovirus-HepG2 system to study the effects of (-)-beta-2', $3^{\prime}$-dideoxy-3'-thiacytidine on replication of hepatitis $B$ virus and accumulation of covalently closed circular DNA. Antimicrob. Agents Chemother. 43, 2017-2026 (1999).

137. Gaillard, R.K. et al. Kinetic analysis of wild-type and YMDD mutant hepatitis B virus polymerases and effects of deoxyribonucleotide concentrations on polymerase activity. Antimicrob. Agents Chemother. 46, 1005-1013 (2002).

138. Abdelhamed, A.M., Kelley, C.M., Miller, T.G., Furman, P.A. \& Isom, H.C. Rebound of hepatitis $B$ virus replication in HepG2 cells after cessation of antiviral treatment. J. Virol. 76, 8148-8160 (2002).

139. Fipaldini, C., Bellei, B. \& La Monica, N. Expression of hepatitis C virus CDNA in human hepatoma cell line mediated by a hybrid baculovirus-HCV vector. Virology 255, 302-311 (1999)

140. McCormick, C.J., Rowlands, D.J . \& Harris, M. Efficient delivery and regulable expression of hepatitis $C$ virus full-length and minigenome constructs in hepatocyte-derived cell lines using baculovirus vectors. J. Gen. Virol. 83, 383-394 (2002).

141. McCormick, C.J., Challinor, L., Macdonald, A., Rowlands, D.J . \& Harris, M. Introduction of replication-competent hepatitis $C$ virus transcripts using a tetracycline-regulable baculovirus delivery system. J. Gen. Virol. 85, 429-439 (2004)

142. Wagle, M. et al. EphrinB2a in the zebrafish retinotectal system. J. Neurobiol. 59 57-65 (2004)

143. Nicholson, L.J ., Philippe, M., Paine, A.J ., Mann, D.A. \& Dolphin, C.T. RNA interference mediated in human primary cells via recombinant baculoviral vector. Mol. Ther 11, 638-644 (2005).

144. Pfohl, J.L. et al. Titration of KATP channel expression in mammalian cells utilizing recombinant baculovirus transduction. Receptors Channels 8, 99-111 (2002).

145. Boudjelal, M., et al. The application of bacmam technology in nuclear receptor drug discovery. Biotechnol. Ann. Rev. 11, in press, (2005).

146. Ames, R. et al. BacMam recombinant baculoviruses in $\mathrm{G}$ protein-coupled receptor drug discovery. Receptors Channels 10, 99-107 (2004).

147. Ames, R. et al. Heterologous expression of $\mathrm{G}$ protein-coupled receptors in $\mathrm{U}-2$ OS osteosarcoma cells. Receptors Channels 10, 117-124 (2004).

148. J enkinson, S. et al. Development of a novel high-throughput surrogate assay to measure HIV envelope/CCR5/CD4-mediated viral/cell fusion using bacmam baculovirus technology. J. Biomol. Screen. 8, 463-470 (2003)

149. Su, J.L. et al. A cell-based time-resolved fluorescence assay for selection of antibody reagents for $\mathrm{G}$ protein-coupled receptor immunohistochemistry. J . Immunol Methods 291, 123-135 (2004).

150. Kost, T.A., Condreay, J .P. \& Mickelson, C.A. Biosafety and viral gene transfer vectors in Biological Safety, Principles and Practices, edn. 3, (eds. Fleming, D.O. \& Hunt D.L.) 579-597 (ASM Press, Washington, DC, 2000). 